syndecan-1 expression. These questions are the subject of ongoing studies into the role of RAGE activation in endothelial dysfunction in SIRS.

\section{S73 CIRCULATING REGULATORS OF ACUTE MUSCLE WASTING IN THE CRITICALLY ILL: GDF-15 A POTENTIAL NOVEL DRIVER OF ACUTE MUSCLE WASTING}

doi:10.1136/thoraxjnl-2012-202678.079

${ }^{1}$ SAA Bloch, ${ }^{1} \mathrm{JY}$ Lee, ${ }^{2} \mathrm{SJ}$ Wort, ${ }^{2} \mathrm{Ml}$ Polkey, ${ }^{1} \mathrm{PR}$ Kemp, ${ }^{2} \mathrm{MJD}$ Griffiths. ' $1 / m p e r i a l$ College, London, UK; ' 2 Department of Respiratory and Critical Care, Royal Brompton Hospital, London, UK

Introduction Acute muscle wasting in the critically ill manifesting as intensive care unit acquired paresis (ICUAP) is common, and causes significant morbidity and mortality. The pathological mechanisms underlying ICUAP are poorly understood and, other than rehabilitation, no therapeutic interventions exist. It was hypothesised that known or potential circulating modulators of muscle mass: insulin-like growth factor-1 (IGF-1), myostatin and growth and differentiation factor-15 (GDF-15) would have distinct dynamic profiles in patients who develop acute muscle wasting in a novel human model of ICUAP.

Methods In a prospective observational study of 42 patients undergoing elective high-risk cardiothoracic surgery (at the Royal Brompton Hospital, London, UK) those who developed muscle wasting were identified by ultrasound. Circulating IGF-1, myostatin and GDF-15 were assayed pre-operatively and over the first week post-operatively. The ability of GDF-15 to cause muscle wasting in vitro was determined in cultured C2C12 myotubes.

Main results 23 of 42 patients (55\%) developed quadriceps atrophy. Plasma concentrations of IGF-1 and unexpectedly myostatin, known mediators of muscle hypertrophy and atrophy respectively, both decreased from baseline in the first post operative days. By contrast, plasma GDF-15 concentrations increased in all patients. This increase in GDF-15 was higher $(535 \% \pm 308 \%$ versus $495 \% \pm 247 \%$ ) and sustained at day 7 in those who developed muscle wasting (day 7 compared with baseline, $p<0.01$ ), but recovered in the non-wasting group ( $p>0.05)$. Furthermore IGF-1 also did not recover in those who developed muscle wasting (day 7 compared with baseline, $p<0.01)$ but did in the non-wasting group $(p>0.05)$.
Myostatin recovered to baseline in both groups. Finally, we demonstrated that GDF-15 caused atrophy of myotubes in vitro (average control myotube diameter was $26.3 \pm 9.0 \mu \mathrm{m}$ versus $22.3 \pm 7.5 \mu \mathrm{m}$ for GDF-15 treated myotubes, equating to $15 \%$ myotube atrophy, $\mathrm{p}=0.011$ ).

Conclusion This data supports the hypothesis that acute muscle loss occurs as a result of an imbalance between drivers of muscle atrophy and hypertrophy. GDF-15 is a potential novel mediator of muscle atrophy in ICUAP, which may become a therapeutic target in patients with ICUAP and other forms of acute muscle wasting.

\section{S74 INCIDENCE AND ANALYSIS OF RISK FACTORS FOR THROMBOCYTOPENIA IN CRITICALLY ILL PATIENTS}

doi:10.1136/thoraxjnl-2012-202678.080

'LK Gupta, ${ }^{2 B}$ James. 'Geisinger Medical Center, Danville, PA, USA; ${ }^{2}$ Regional Medical Center of San Jose, San Jose, CA, USA

Introduction Thrombocytopenia occurs in 15\% to $58 \%$ of intensive care unit patients. Up to $25 \%$ of acutely ill patients develop drug-induced thrombocytopenia. Establishing the etiology of secondary thrombocytopenia is challenging, since many medications and co morbidities have been implicated.

Objective To define the incidence and severity of thrombocytopenia in critically ill patients and to examine risk factors that may be related to the development of thrombocytopenia.

Method This is a retrospective chart review of 328 critically ill patients with thrombocytopenia requiring at least 72 hours of critical care. 30 suspected risk factors, including patient characteristics, drug effects, and associated diseases were identified from the literature and further evaluated using a multiple logistic regression. Mild Thrombocytopenia defined as platelet count less than $200 \times 10$ (9)/L was observed frequently, but only 9 percent had severe thrombocytopenia defined as less than $20 \times 10(9) / L$.

Results Demographic characteristics of patients predisposed to thrombocytopenia are males, older than 55, and Hispanic population. Hemodynamic instability and/or heparin exposure appear to be the strongest identifiable correlates with thrombocytopenia. Sepsis, acute kidney injury and respiratory failure were strongly correlated $(p<0.0001)$ with thrombocytopenia. Patients with severe thrombocytopenia were found to have concurrent alterations in

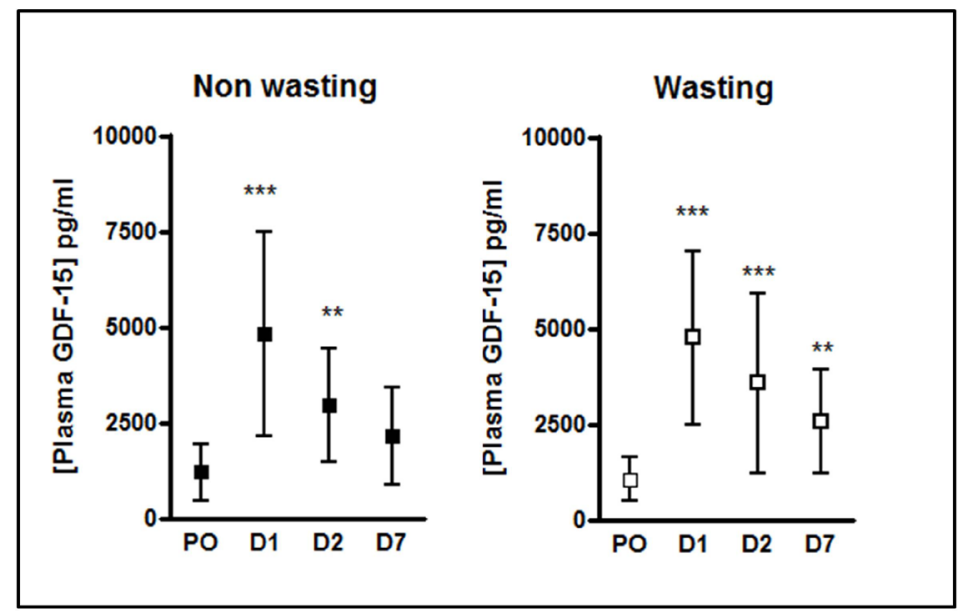

Plasma GDF-15 concentration $(\mathrm{pg} / \mathrm{ml})$ in non-wasting $(\mathrm{n}=19)$ and wasting patients (those with $>9.24 \%$ muscle loss: $n=23$ ) pre-operatively (PO), on day 1 (D1), day 2 (D2) and on day 7 (D7). Data presented as mean $\pm S D$. ${ }^{*} p<0.01, * * * p<0.001$, repeated measures ANOVA with Bonferroni correction for comparison with pre-operative baseline.

Abstract S73 Figure 1 
liver function tests. Patients with central line placement and heparin exposure were associated strongly with mild thrombocytopenia $(p<0.0001)$. Drug therapies that were correlated with thrombocytopenia included Heparin, Protonix, Lasix, Ativan and Zofran and antibiotics such as Vancomycin, Cephalosporins and Levaquin.

Conclusion Drug regimens should be evaluated daily for minimization of adverse drug events including thrombocytopenia. Once the diagnosis is suspected, clinicians should identify the medication and/or risk factors causing secondary thrombocytopenia to assess the timeline of development. Co morbidity associated with thrombocytopenia was sepsis syndrome, liver disorder, alcoholism and atrial fibrillation. Medications commonly associated with druginduced thrombocytopenia include glycoprotein IIb/IIIa inhibitors, cinchona alkaloids, antibiotics, anticonvulsants, and heparin. Thrombocytopenia generally resolved in most patients with critical management of the disease and discontinuation of the offending medication.

\section{S75 HIGH DOSE VITAMIN D SUPPLEMENTATION IMPROVES EXTRAVASCULAR LUNG WATER INDEX AND IN-VIVO TREG AND LL37 RESPONSES POST-0ESOPHAGECTOMY}

doi:10.1136/thoraxjnl-2012-202678.081

'RCA Dancer, 'D Parekh, ${ }^{2} \mathrm{AR}$ Martineau, ${ }^{3} \mathrm{GD}$ Perkins, 'DR Thickett. 'University of Birmingham, Birmingham, UK; ${ }^{2}$ Bartsthe London School of Medicine and Dentistry, London, UK; ${ }^{3}$ Warwick Medical School, Coventry, UK

Acute Lung Injury occurs in around $25 \%$ of patients post 2 stage oesophagectomy. We have previously shown that levels of $25-\mathrm{OH}$ vitamin $\mathrm{D}$ are low in these patients and that those patients with the lowest levels of vitamin D are more likely to develop Acute Lung Injury post-operatively. In vitro, vitamin $\mathrm{D}$ has been shown to promote the differentiation of regulatory $\mathrm{T}$ cells (Treg) and the expression of LL37 (cathelicidin), an antimicrobial peptide.

We have given 13 patients a one-off high dose vitamin $\mathrm{D}$ supplement 3-14 days prior to oesophagectomy. Numbers of circulating regulatory $\mathrm{T}$ cells were analysed both pre-and post-supplementation. Plasma levels of LL37 were measured by ELISA. Extravascular Lung Water Index (EVLWi) was measured pre-operatively, post-operatively and on the day following surgery. Changes in LL37 and EVLWi were compared with results from a cohort of patients $(n=50)$ who had not been supplemented.

Pre-operative vitamin D levels between the two groups were significantly different. The proportion of CD3 $+\mathrm{CD} 4+\mathrm{T}$ cells which were CD25+CD127loFoxP3+ increased post vitamin D supplementation (median pre vitamin $\mathrm{D}=5.8$, median post vitamin $\mathrm{D}=7.3, \mathrm{p}=0.028$ ). Whilst levels of LL37 decreased post-operatively in the cohort who had not received vitamin $\mathrm{D}$, levels were maintained in supplemented patients. Post-operative EVLWi measured lower than pre-operative values in patients receiving vitamin $\mathrm{D}$ supplementation. This contrasts with patients who did not receive vitamin $\mathrm{D}$ supplementation in whom an increase in EVLWi was seen. This difference persists on post-operative day 1, with a net increase being seen in patients who did not receive vitamin $\mathrm{D}$ and a net decrease in those who did (see table).

Conclusions A stat high dose vitamin D supplement restored vitamin D levels in the week before oesophagectomy. This was associated with elevated circulating Treg levels in vivo and an increase in post-operative plasma LL37 expression suggesting the vitamin D supplementation was biologically active.

Patients receiving vitamin $\mathrm{D}$ had no perioperative increase in extravascular lung water compared to our historical cohort suggesting that vitamin $\mathrm{D}$ protects against alveolar epithelial damage perhaps in part due to effects on circulating Treg cells and anti-microbial peptide production.
Abstract S75 Table 1 Effects of Vitamin D supplementation on median values of Vitamin D, EVLWi and LL37

\begin{tabular}{lccc}
\hline & $\begin{array}{l}\text { Vitamin D } \\
\text { supplementation }\end{array}$ & $\begin{array}{l}\text { No Vitamin D } \\
\text { supplementation }\end{array}$ & $\begin{array}{l}\text { p value } \\
\text { (Mann-Whitney U test) }\end{array}$ \\
\hline 25-OH Vitamin D level & $76 \mathrm{nmol} / \mathrm{L}$ & $25 \mathrm{nmol} / \mathrm{L}$ & 0.001 \\
Change in EVLWi D0 post-op & -1.0 & 2.0 & 0.004 \\
Change in EVLWi D1 post-op & -1.0 & 1.0 & 0.023 \\
\% change in plasma LL37 as & 10.9 & -17.8 & 0.003 \\
proportion of total protein & & & \\
post-op & & & \\
\hline
\end{tabular}

\section{S76 PROTEOLYTIC CLEAVAGE OF ELAFIN BY 20S PROTEASOME MAY CONTRIBUTE TO INFLAMMATION IN ACUTE LUNG INJURY}

doi:10.1136/thoraxjnl-2012-202678.082

${ }^{1}$ A Kerrin, 'S Weldon, ${ }^{2} \mathrm{~A}$ Chang, 'T Craig, ${ }^{3} \mathrm{AJ}$ Simpson, 'C O'Kane, 'DF McAuley, ${ }^{1} \mathrm{C}$ Taggart. Queen's. 'University Belfast, Belfast, United Kingdom; 'National Institutes of Health, Bethesda, USA; ${ }^{3}$ University of Newcastle, Newcastle, united Kingdom

The Aim of this study was to characterise temporal changes in elafin concentration in patients with acute lung injury (ALI) and to evaluate whether a decrease in elafin levels is due to elevated protease activity. Previous work has shown that unregulated protease activity can cause proteolytic cleavage of elafin, impairing the innate immune function of the protein. Bronchoalveolar lavage fluid (BALF) was obtained from patients with ALI within 48 hours of onset of ALI (day 0), at day 3 and at day 7. Elafin levels were quantified by ELISA Elafin susceptibility to proteolytic cleavage by ALI BALF was assessed by Western blot and by HPLC-Mass Spectrometry. Elafin levels were found to be significantly increased at the onset of ALI compared to healthy volunteers and fell significantly by day 7 compared to day 0 . In contrast, levels of secretory leukocyte protease inhibitor (SLPI) did not decrease over time. This decrease in elafin was due to cleavage by the $20 \mathrm{~S}$ proteasome which was significantly increased in ALI BALF. Incubation of ALI BALF with the proteasome inhibitor epoxomicin confirmed that $20 \mathrm{~S}$ proteasome protease activity was responsible for proteolytic cleavage of elafin resulting in diminished anti-elastase activity. In addition, free neutrophil elastase (NE) activity significantly increased in ALI BALF from day 0 to day 7. In conclusion, elafin concentrations decrease within the pulmonary compartment over the course of ALI as a result of proteolytic degradation. This loss of elafin may predispose, in part, to excessive inflammation in ALI.

\section{S77 PRONE POSITIONING AND INTRAVENOUS STEROIDS FOR THE MANAGEMENT OF SEVERE ARDS}

doi:10.1136/thoraxjnl-2012-202678.083

L Gupta, Ben SiuJames. Regional Medical Center of San Jose, San Jose, USA

Introduction Prone positioning and intravenous steroids represents a treatment option in patients with severe ARDS because most of the clinical manifestations in ARDS is secondary to excess fluid in the interstitium and alveoli and presence of inflammatory cells in the fluid.

Objective To assess the impact of prone positioning and steroids in severe ARDS and to assess and identify prognostic factors and potential predictors of mortality in ARDS patients.

Methods This is a retrospective study of 46 patients with severe ARDS from 2009-2011. The patient charts were reviewed and the impact of prone positioning and steroids was observed on severe ARDS patients. The primary outcome measured were ICU length of stay, number of ventilator days and 30 day mortality. 